\title{
Free vibration analysis of a composite beam with single delamination- An improved free and constrained model
}

\author{
M. Shariati Nia ${ }^{a}$ K. Torabi ${ }^{\mathrm{b}}$ and M. Heidari-Rarani ${ }^{\mathrm{c}^{*}}$
}

${ }^{a}$ Ph.D. Student, University of Kashan, Kashan 8731751167, Iran

${ }^{b}$ Assosiate Professor, University of Kashan, Kashan 8731751167, Iran

${ }^{c}$ Assistant Professor, Department of Mechanical Engineering, Faculty of Engineering, University of Isfahan, Isfahan, 8174673441, Iran

\section{A R T I C L E I N F O}

\section{Article history:}

Received June 6, 2014

Accepted 24 July 2014

Available online

24 July 2014

Keywords:

Free vibration

Composite beams

Delamination

Natural Frequency

\begin{abstract}
A B S T R A C T
In this paper, an analytical method is proposed for calculation of natural frequencies of a delaminated composite beam from both free and constrained mode frequencies. In previous studies, the frequencies of a delaminated composite beam were computed with assumption of occurring open or close delamination during the vibration. According to this assumption, two separated modes, i.e., "free mode" and "constrained mode", are occurred in vibration of the delaminated beam. In fact, a delamination may breathe (open and close) during the vibration and the assumptions of the free or constrained mode models are not completely correct in the whole of the vibration period. For this reason, a new formulation is proposed for calculation of natural frequencies based on the breathing of delamination. The obtained results are compared with various theoretical and experimental results available in the literature. Thus, the effects of location and size of delamination can be investigated on the natural frequencies of delaminated beams.
\end{abstract}

\section{Introduction}

Advanced composite materials are increasingly used in structural designs of aircraft, helicopters, spacecraft, automobiles, marine and submarine vehicles because of desirable properties like high strength and stiffness, lightweight, fatigue resistance, and damage tolerance, etc. However, composites are very sensitive to the anomalies induced during their fabrication or service life. One of the commonly encountered types of defects or damage in laminated composite structures is delamination. Delaminations may originate during fabrication or may be service-induced, such as by impact of fatigue loading. Delaminations not only affect the strength and integrity of the structure but also cause the reduction of the stiffness, thus affecting its vibration and stability characteristics. Reflections of these effects in dynamic response are the alteration of natural frequencies and damping ratios. In addition "delamination modes" which are related to the opening of the delaminated region during the dynamic response will appear in some cases. As a result, considerable analytical, numerical and experimental efforts have been expended to capture these phenomena.

\footnotetext{
* Corresponding author.

E-mail addresses: m.heidarirarani@eng.ui.ac.ir (M. Heidari-Rarani)
} 
One of the earliest models for vibration analysis of composite beams including delaminations was proposed by Ramkumar et al. (1979). They modeled a beam with one through-thickness delamination by simply using four Timoshenko beams connected at delamination edges. Natural frequencies and mode shapes were solved by a boundary eigenvalue problem. By using their model, the predicted natural frequencies were consistently lower than the results reported in experimental measurements. Authors attributed this discrepancy to the effect of contact between the delaminated "free" surfaces during vibrations. They suggested that the inclusion of the contact effect may improve the analytical prediction.

To study the influence of a through-width delamination on the free vibration of an isotropic beam, Wang et al. (1982) presented an analytical model using four Euler-Bernoulli beams that are joined together. They assumed that the delaminated layers deform "freely" without touching each other ("free mode" model) and will have different transverse deformations. Later, Mujumdar and Suryanarayan (1988) presented two models namely the free mode model and the constrained mode model for the flexural vibrations of isotropic beams with delamination at the mid-plane as well as at the off-mid-plane locations. Experimental results have also been presented for various cases of delaminations in the beams. The paper concluded that the constrained mode model in which the transverse displacement and the normal stress of the upper and lower layer at the delaminated interface have been constrained to be the same, gives results in good agreement with the experimental results. The free mode model (similar to the one developed by Wang) under-estimates the frequencies, particularly at the higher modes.

The "constrained model" fails to explain the delamination opening modes found in experiments. In these experiments conducted by Shen and Grady (1992), opening modes were even found in the first bending mode of the beam for some delamination cases. However, their finite element formulations (Model A and B) were essentially followed the "constrained model" by Mujumdar and Suryanarayan (1988) and the "free model" by Wang et al. (1982). In this paper "Model A" is corresponding to the "constrained model" and the "Model B" is corresponding to the "free model". The discrepancy between the results predicted by the two models is significant even in cases where mode shapes do not show any opening in the delamination region. Furthermore, in some cases, opening delamination modes were shown clearly in their experiment, while the "constrained model" frequency prediction had a better match with the corresponding experimental results for these modes, even though the delamination cannot open using the "constrained model".

Hu and Hwu (1995) presented an analytical model based on the Timoshenko beam theory for the free vibrations of delaminated sandwich beams. Lee (2000) investigated a displacement-based layerwise finite element model for the analysis of free vibration of delaminated beam. in this study, the effects of the fiber angle, location, size and number of delamination are investigated. A review paper on the vibration-based model-dependent damage (delamination) identification and health monitoring for composite structures is presented by Zou et al. (2000). This paper deals with various models proposed for the free vibrations of delaminated beams. In the analytical research done by Luo and Hanagud (2000), a piecewise-linear spring model is used to simulate the behavior between delaminated surfaces. Shear and rotary inertia effects, as well as bending-extension coupling, are included in the governing equations. Frequencies and mode shapes are solved through a boundary eigenvalue problem. The proposed model includes the "free model" and the "constrained model" as special cases. The nonlinear response simulated by this model shows good agreement with the experiment results.

Karmakar et al. (2005) presented the effect of delamination on free vibration characteristics of graphite-epoxy composite pre-twisted shallow shells of various stacking sequences considering length of delamination as a parameter. An exhaustive review on the vibration of delaminated composites has been presented by Della et al. (2007). The paper deals with various analytical models 
and numerical analysis for the free vibration of composite laminates. Della et al. (2009) developed analytical solutions to study the free vibrations of multiple delaminated beams under axial compressive loadings. The Euler-Bernoulli beam theory and free mode and constrained mode assumption in delamination buckling and vibration are used in the analysis. Ramtekkar (2009) presented free vibration analysis of laminated beams with delamination using mixed finite element model. Analytical solutions for beams with multiple delaminations have been presented by some researchers. Shu and Della (2004, 2004) and Della and Shu (2005) used the "free mode" and "constrained mode" assumptions to study a composite beam with various multiple delamination configurations. Their study emphasized on the influence of a second delamination on the first and second natural frequencies and the corresponding mode shapes of a delaminated beam. Della and Shu (2007) also studied the free vibration of a delaminated bimaterial beam using Euler-Bernoulli beam theory.

In this paper, an improved analytical formulation is proposed for calculation of combined natural frequencies based on the free and constrained mode frequencies derived from theoretical methods. This analysis has been conducted by employing the "free mode" and the "constrained mode" models on the beam with single delamination with various lengths and depth (different interfaces) located at the center of the beam. The improved combined natural frequencies have been considered and the results have been compared with various theoretical and experimental results available in the literature. These results address the effects of the location and size of delamination on natural frequencies of delaminated beams.

\section{Theoretical modeling}

Fig. 1 shows a cantilever beam with a single delamination at an arbitrary location and various lengths. $L, h$ and $a$ are beam length, beam thickness and delamination length, respectively.

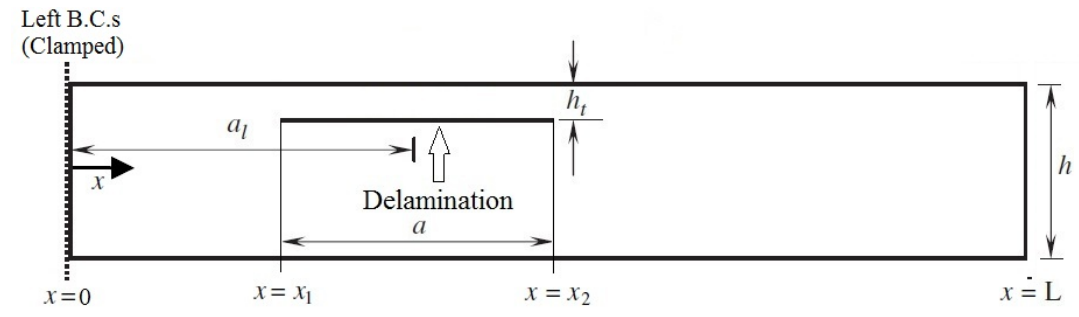

Fig 1. Geometry of the delaminated beam

The beam is separated along the interface by a delamination with length $a$ and located at the center of the beam. Dimensionless depth of delamination is defined as:

$\alpha_{t}=h_{t} /(h / 2)$,

where $h_{t}$ is the depth of delamination from the top of the beam. Small values of $\alpha_{t}$ refers to delamination nearer to top surface of beam and $\alpha_{t}=1$ refers to mid-plane delamination $\left(h_{t}=h / 2\right)$.

The beam can be subdivided into three span-wise regions, a delamination region and two integral regions. The delamination region is included of two segments (delaminated layers), beam 2 and beam 3 , which are joined at their ends to the integral segments, beam 1 and beam 4 . Each of the four beams is treated as beams with different boundary conditions.

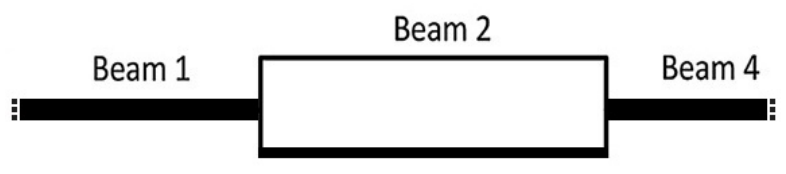

Beam 3

Fig 2. Delaminated beam as four interconnected beams 


\subsection{Free mode model}

In "free mode" model, it is assumed that the delaminated layers deform "freely" without touching each other and have different transverse deformations. The governing equations for the free vibration of a delaminated beam using the Euler-Bernoulli beam theory are:

$D_{i} \frac{\partial^{4} w_{i}}{\partial x^{4}}+\rho_{i} A_{i} \frac{\partial^{2} w_{i}}{\partial t^{2}}=0, \quad i=1,2,3,4$

where $D_{i}$ is the reduced bending stiffness of the $i$ th beam and is presented by Shu and Della (2004), $\rho_{i}$ is the mass density and $A_{i}$ is the cross-sectional area of the beam. The mechanical properties of the composite beams are determined using the classical laminate theory. The bending stiffness for homogeneous and isotropic beams is given by $D_{i}=E I_{i}$, where $\mathrm{E}$ denote the Young's modulus and $I$ is the moment of inertia. For free vibration

$w_{i}\left(x_{i}, t\right)=W_{i}\left(x_{i}\right) \mathrm{e}^{\mathrm{i} \omega t}$

where $\omega$ is the natural frequency and $W_{i}$ is the mode shape. Substituting Eq. (3) into Eq. (2) and eliminating the trivial solution $\sin (\omega t)=0$, one can obtain the general solutions of the differential equation in Eq. (2) as

$W_{i}(x)=C_{i} \cos \left(\lambda_{i} x\right)+S_{i} \sin \left(\lambda_{i} x\right)+C H_{i} \cosh \left(\lambda_{i} x\right)+S H_{i} \sinh \left(\lambda_{i} x\right)$

where

$\lambda_{i}^{4}=\frac{\omega^{2} \rho_{i} A_{i}}{D_{i}}$

and $\lambda_{i}$ are the non-dimensional frequencies. The lowest eigenvalue $\lambda$ is the non-dimensional primary frequency of the beam. The 16 unknown coefficients $C_{i}, S_{i}, \mathrm{CH}_{i}$ and $\mathrm{SH}_{i}$ are determined by four boundary conditions and twelve continuity conditions (Shu \& Della, 2004). A non-trivial solution for the coefficients exists only when the determinant of the coefficient matrix vanishes. The frequencies and mode shapes can be obtained as eigenvalues and eigenvectors, respectively.

\subsection{Constrained mode model}

The "constrained mode" model is simplified by the assumption that the delaminated layers are in touch along their whole length all the time, but are allowed to slide over each other. Therefore, the delaminated layers have the same transverse deformations $\left(w_{3}=w_{2}\right)$. This is reasonable since if there is no opening in the delamination region, the "free model" and "constrained model" are essentially the same. In this model the governing equations are:

$D_{i} \frac{\partial^{4} w_{i}}{\partial x^{4}}+\rho_{i} A_{i} \frac{\partial^{2} w_{i}}{\partial t^{2}}=0 \quad i=1,4$

For beams 2 and 3;

$\left(D_{2}+D_{3}\right) \frac{\partial^{4} w_{2}}{\partial x^{4}}+\left(\rho_{2} A_{2}+\rho_{3} A_{3}\right) \frac{\partial^{2} w_{2}}{\partial t^{2}}=0$

The generalized solutions for the constrained model are identical in form to the free model. The unknown coefficients $C_{i}, S_{i}, C H_{i}$ and $S H_{i}$, however, are reduced to twelve coefficients, which can be determined by four boundary conditions and eight continuity conditions.

\subsection{Combined natural frequency}

Here, an example is presented for calculation of combined natural frequency from Lee (2000). In this example, a delamination is located close to the surface $\left(h_{t} / h=0.2\right)$ as shown in Fig. 1 . For $a / L=$ 0.3, the calculated first vibration mode shape is demonstrated in Fig. 3(a). As shown in this figure, 
the delamination opens when the beam vibrates upward. When the beam vibrates downward, however, this mode shape is inadequate for representing the motion because there may be an overlapping between two sublaminates. In fact, the delamination should close when the beam goes down (Fig. 3(c)). Consequently, the beam does not exhibit classical vibration modes, and strictly speaking, the natural frequencies cannot be defined in this case. However, assuming that the two delaminated parts have the same velocities as well as same deflections at status $(b)$ in Fig. 3, a total period $T$ can be approximated by averaging of the period of two states as follows:

$T=\frac{1}{2}\left[T_{\text {open (free) }}+T_{\text {closed (constrained) }}\right]$

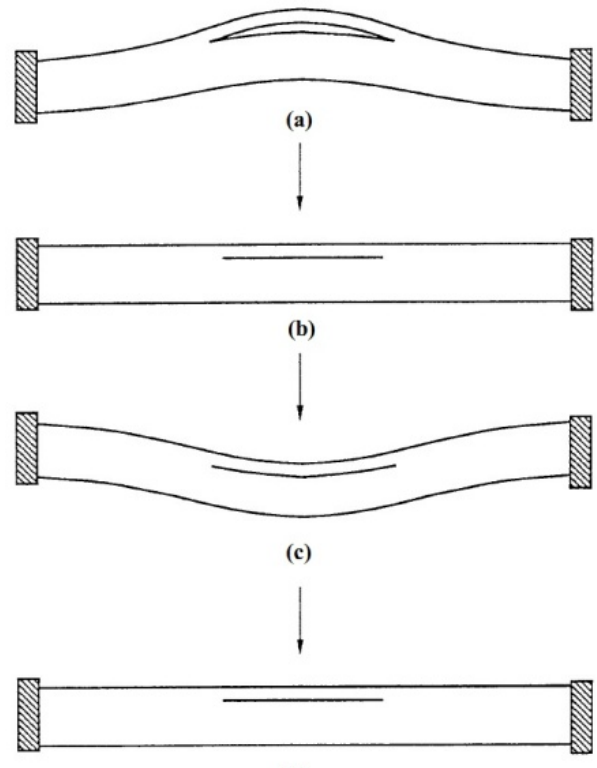

(d)

Fig 3. Vibration mode change during the period of the motion for a clamped beam with $h_{t} / h=0.2$, $a / L=0.3$ (Lee, 2000)

Accordingly, from the relation between period and natural frequency, the combined natural frequencies can be estimated as follows:

$\omega=\frac{2 \omega_{\text {free }} * \omega_{\text {constrained }}}{\omega_{\text {free }}+\omega_{\text {constrained }}}$

The first non-dimensional natural frequencies for the modes of open delamination, closed delamination, and combined modes are presented for $a / L=0.3$ and 0.6 in Table 1 . It should be noted that the velocity matching assumption appears to be somewhat inappropriate for a longer delamination $(a / L=0.6)$. This is because the first vibration mode for delamination opening shows almost pure local vibration of the thin upper layer while delamination opening-suppressed mode is global. This results in substantial difference in vibration frequencies between the two cases. Therefore, more rigorous analysis appears to be necessary for local-mode type vibration of delaminated composites.

Table 1. Natural frequencies of a clamped beam with $h_{t} / h=0.2$

\begin{tabular}{lll}
\hline Delamination length, $(a / L)$ & 0.3 & 0.6 \\
\hline Open delamination mode & 22.08 & 12.05 \\
Close delamination mode & 22.32 & 21.29 \\
Combined mode & 22.20 & 15.39 \\
\hline
\end{tabular}




\section{Improved combined natural frequency}

In this paper, a new improved analytical formulation is introduced for calculation of combined natural frequency based on the free and constrained frequencies. In this method, total period $T$ is approximated by adding the period of two states with weighted factors as follows:

$T=\alpha_{t} T_{\text {free }}+\left(1-\alpha_{t}\right) T_{\text {constrained }}$

where $\alpha_{t}$ is defined in Eq. (1). Accordingly, from the relation between period and natural frequency, the combined natural frequencies can be estimated as follows:

$\omega=\frac{\omega_{\text {free }} * \omega_{\text {constrained }}}{\alpha_{t} \omega_{\text {free }}+\left(1-\alpha_{t}\right) \omega_{\text {constrained }}}$

The obtained results from the proposed method are compared with various theoretical and experimental results available in the literature. This results and comparison also addresses the effects of the location and size of delamination on natural frequencies of delaminated beams.

Numerical investigations are undertaken for the free vibrations of cross-ply laminated cantilevered beams with embedded internal delaminations of different sizes and at several different locations. For this purpose, an eight-layer symmetric cross-ply laminated $[0 / 90]_{2 s}$ beam, under the clamped-free supports is considered. The locations of delaminations through-the-thickness are defined as mentioned in the work of Shen and Grady (1992). "Interface 1" implies the mid-plane delamination, while "Interface 4" implies the skin ply delamination and so on. Lengthwise delamination locations are at the middle of beams. Delamination dimensionless length $(a / L)$ includes 0 (intact), 0.2, 0.4, 0.6, and 0.8. Fig. 3 shows interfaces and dimensions for a cross-ply delaminated beam. The mechanical properties of the unidirectional graphite/epoxy are presented in Table 2.

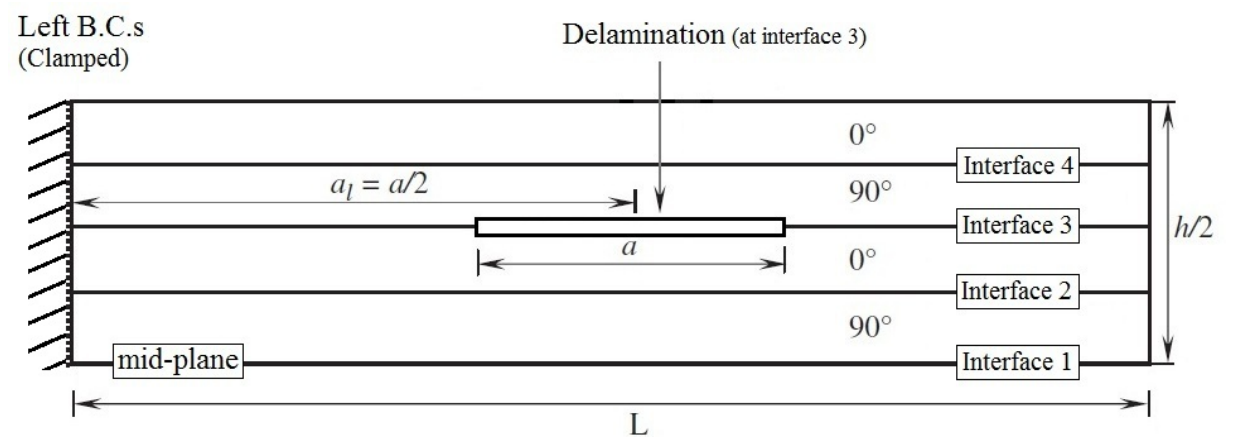

Fig 3. Geometry of the eight layer symmetric composite laminated beam $[0 / 90]_{2 \mathrm{~s}}$

Table 2. Mechanical properties of the unidirectional graphite/epoxy (Ramtekkar, 2009)

\begin{tabular}{ccccc}
\hline$E_{11}, \mathrm{GPa}$ & $E_{22}, \mathrm{GPa}$ & $G_{12}, \mathrm{GPa}$ & $v_{12}$ & $\rho, \mathrm{kg} / \mathrm{m}^{3}$ \\
\hline 134.49 & 10.34 & 5 & 0.3 & 1477.60 \\
\hline
\end{tabular}

\section{Results and Discussion}

The fundamental frequencies of a delaminated composite beam with various locations and sizes of delamination are presented in Table 3-6. The experimental results and analytical results related to Model A and B are provided from Shen and Grady (1992). The frequencies obtained from the combined natural frequency model are presented by Lee (2000). Improved natural frequencies obtained from new formulation presented in this paper are also presented in the tables. It is observed that the fundamental frequencies obtained by the new improved analytical formulation for calculation of combined natural frequency, closely match with the experimental results of Shen and Grady (1992) for most of the cases. 
Table 3. Fundamental natural frequencies (Hz) for delamination along interface 1

\begin{tabular}{llccccc}
\hline & Delamination length $(a / L)$ & 0.0 & 0.2 & 0.4 & 0.6 & 0.8 \\
\hline \multirow{2}{*}{$\begin{array}{l}\text { Shen and } \\
\text { Grady (1992) }\end{array}$} & Experiment (average test) & 79.833 & 78.167 & 75.369 & 67.959 & 57.542 \\
& Analytical model A (constrained model) & 82.042 & 80.133 & 75.285 & 66.936 & 57.239 \\
& Analytical model B (free model) & 82.042 & 67.363 & 56.479 & 47.898 & 40.586 \\
\hline Lee (2000) & Combined natural frequency & 82.042 & 73.195 & 64.540 & 55.839 & 47.495 \\
\hline Present study & Improved combined natural frequency & 82.042 & 80.133 & 75.285 & 66.936 & 57.239 \\
\hline
\end{tabular}

Table 4. Fundamental natural frequencies $(\mathrm{Hz})$ for delamination along interface 2

\begin{tabular}{llccccc}
\hline & Delamination length $(a / L)$ & 0.0 & 0.2 & 0.4 & 0.6 & 0.8 \\
\hline \multirow{2}{*}{$\begin{array}{l}\text { Shen and } \\
\text { Grady (1992) }\end{array}$} & Experiment (average test) & 79.833 & 77.792 & 75.126 & 66.958 & 48.335 \\
& Analytical model A (constrained model) & 82.042 & 81.385 & 78.103 & 71.159 & 62.121 \\
& Analytical model B (free model) & 82.042 & 68.776 & 59.438 & 51.180 & 43.860 \\
\hline Lee (2000) & Combined natural frequency & 82.042 & 74.551 & 67.504 & 59.538 & 51.417 \\
\hline Present study & Improved combined natural frequency & 82.042 & 77.818 & 72.418 & 64.832 & 56.265 \\
\hline
\end{tabular}

Table 5. Fundamental natural frequencies (Hz) for delamination along interface 3

\begin{tabular}{llccccc}
\hline & Delamination length $(a / L)$ & 0.0 & 0.2 & 0.4 & 0.6 & 0.8 \\
\hline \multirow{2}{*}{$\begin{array}{l}\text { Shen and } \\
\text { Grady (1992) }\end{array}$} & Experiment (average test) & 0.0 & 0.2 & 0.4 & 0.6 & 0.8 \\
& Analytical model A (constrained model) & 79.833 & 80.125 & 79.750 & 76.958 & 72.460 \\
\cline { 2 - 6 } & Analytical model B (free model) & 82.042 & 81.461 & 79.932 & 76.712 & 71.663 \\
\hline Lee (2000) & Combined natural frequency & 82.042 & 75.137 & 70.416 & 65.058 & 59.131 \\
\hline Present study & Improved combined natural frequency & 82.042 & 78.171 & 74.873 & 70.406 & 64.797 \\
\hline
\end{tabular}

Table 6. Fundamental natural frequencies $(\mathrm{Hz})$ for delamination along interface 4

\begin{tabular}{llccccc}
\hline & Delamination length $(a / L)$ & 0.0 & 0.2 & 0.4 & 0.6 & 0.8 \\
\hline \multirow{2}{*}{ Shen and } & Experiment (average test) & 79.833 & 79.958 & 68.917 & 62.500 & 55.626 \\
Grady (1992) & Analytical model A (constrained model) & 82.042 & 81.598 & 80.383 & 77.698 & 73.147 \\
& Analytical model B (free model) & 82.042 & 75.834 & 71.881 & 67.181 & 61.704 \\
\hline Lee (2000) & Combined natural frequency & 82.042 & 78.610 & 75.895 & 72.058 & 66.940 \\
\hline Present study & Improved combined natural frequency & 82.042 & 77.197 & 73.833 & 69.534 & 64.215 \\
\hline
\end{tabular}

\section{Conclusions}

Free vibration behavior of delaminated composite beams with different stacking sequences and various delamination sizes are investigated in this study. An improved analytical formulation is oriented for calculation of combined natural frequency based on the free and constrained modes derived from theoretical methods. The frequencies obtained from the proposed model are assessed by various theoretical and experimental results available in the literature. These results address the effects of the location and size of delamination on natural frequencies of delaminated beams. The proposed combined natural frequency is compared with experimental results and shows better agreement than previous formulations for combined natural frequency. The comparison of improved combined natural frequency with theoretical and experimental results shows better agreement.

\section{References}

Della, C.N., \& Shu, D. (2005). Free vibration analysis of composite beams with overlapping delaminations. European Journal of Mechanics-A/Solids, 24(3), 491-503.

Della, C. N., \& Shu, D. (2007). Free vibration analysis of delaminated bimaterial beams. Composite Structures, 80(2), 212-220.

Della, C. N., \& Shu, D. (2007). Vibration of delaminated composite laminates: A review. Applied Mechanics Reviews, 60(1), 1-20.

Della, C. N., \& Shu, D. (2009). Free vibration analysis of multiple delaminated beams under axial compressive load. Journal of Reinforced Plastics and Composites, 28(11), 1365-1381. 
Hu, J.S. \& Hwu, C. (1995). Free vibration of delaminated composite sandwich beams. AIAA Journal, 33(10), 1911-1918.

Karmakar, A., Roy, H. \& Kishimoto, K. (2005). Free vibration analysis of delaminated composite pre-twisted shells, Aircraft Engineering and Aerospace Technology, 77(6), 486-490.

Lee, J. (2000). Free vibration analysis of delaminated composite beams. Computers and Structures, $74,121-129$.

Luo, H., \& Hanagud, S. (2000). Dynamics of delaminated beams. International Journal of Solids and Structures, 37, 1501-1519.

Mujumdar, P.M., \& Suryanarayan, S. (1988). Flexural vibrations of beams with delaminations. Journal of Sound and Vibration, 125 (3), 441-461.

Ramtekkar, G.S. (2009). Free vibration analysis of delaminated beams using mixed finite element model. Journal of Sound and Vibration, 328, 428-440.

Ramkumar, R.L., Kulkarni, S.V., \& Pipes, R.B. (1979). Free vibration frequencies of a delaminated beam. 34th Annual Technical Conference, Reinforced Plastics/Composites Institute. The Society of the Plastics Industry Inc., 1-5.

Shen, M.-H.H., \& Grady, J.E. (1992). Free vibrations of delaminated beams. AIAA Journal, 30(5), 1361-1370.

Shu, D., \& Della, C.N. (2004). Free vibration analysis of composite beams with two non-overlapping delaminations. International Journal of Mechanical Sciences, 46(4), 509-526.

Shu, D., \& Della, C.N. (2004). Vibrations of multiple delaminated beams. Composite Structures, 64(3-4), 467-77.

Wang, J.T.S., Liu, Y.Y., \& Gibby, J.A. (1982). Vibration of split beams. Journal of Sound and Vibrations, 84(4), 491-502.

Zou, Y., Tong, L., \& Steven G.P. (2000). Vibration-based model-dependent damage (delamination) identification and health monitoring for composite structures- A review. Journal of Sound and Vibration, 230(2), 357-378. 